\title{
La identidad después de la gentrificación
}

\author{
Carla Nóbile / Andrea Sader
}

Carla Nóbile: Arquitecta, Facultad de Arquitectura, Universidad ORT Uruguay.

Actividad profesional independiente.

Profesora ayudante de Historia de la Arquitectura.

Andrea Sader: Arquitecta, Facultad de Arquitectura, Universidad ORT Uruguay.

Actividad profesional independiente.

Profesora ayudante de Historia de la Arquitectura. 
La ciudad de Colonia del Sacramento, luego de haber sido incluida en la lista de obras consideradas Patrimonio Histórico de la Humanidad por parte de la UNESCO, (1995) ha experimentado, al igual que otros centros históricos, un resurgir que la ha posicionado como uno de los puntos de mayor valor patrimonial y turístico dentro de la región.

La ciudad experimenta un proceso de transformación, tanto física como social, asociado a los procesos de gentrificación que han pautado las características de la ciudad contemporánea.

Parece interesante la influencia de estos procesos en lo que es la identidad de la ciudad y sus habitantes, temática que da lugar a este artículo.

En el siguiente documento se plantea el estudio del fenómeno de gentrificación, sus características y consecuencias, para luego asociarlo a un caso específico de clara referencia en la región como es Colonia del Sacramento, y así centrarse en la discusión, polémica claramente, de las relaciones identitarias que se suceden en la ciudad actual como consecuencia del proceso anteriormente mencionado.

Como punto de partida para esta publicación se propone una hipótesis que refiere a las transformaciones que ha experimentado, tanto sociales, urbanas y económicas la ciudad de Colonia del Sacramento, una vez incorporada a la lista de la UNESCO como sitio de interés patrimonial.

Si bien en algunos aspectos parece exagerado tratar el caso de Colonia del Sacramento como un claro caso de gentrificación, se intenta dejar en evidencia que más allá de las diferentes escalas, este fenómeno tiene una clara influencia en lo que significa el concepto de ciudad, asociado a los procesos de transformación propios de la realidad contemporánea.

El interés específico en el tema identitario hace referencia a la trascendencia nacional e internacional, que ha adquirido esta ciudad y a cómo esto influye directamente no sólo en los aspectos físicos sino también en los aspectos económicos, pero sobre todo en los aspectos sociales, todos ellos claramente ligados al proceso de gentrificación.

Se plantea que la transformación de identidad en el centro histórico de Colonia del Sacramento se da de forma similar a lo sucedido en otras ciudades contemporáneas, más allá del reconocimiento del valor histórico, más allá de la promoción de intervenciones de conservación y rehabilitación, y por sobre todo, más allá de la participación de los propios habitantes de la ciudad en la recuperación del mismo. 


\section{Gentrificación:}

Conceptos, evolución e involucrados

A mediados de la década del 60 , surge el término "Gentrificación" aplicado por la socióloga británica Ruth Glass ${ }^{1}$ para referirse a los cambios que se observaban en algunos distritos londinenses como Islington donde los pobladores de clases obreras fueron lentamente desplazados por clases sociales más elevadas que, una vez que los contratos de alquiler de aquéllos habían expirado, transformaban esas humildes viviendas en residencias costosas y elegantes.

En una primera etapa, a finales de la década de 1960 y mediados de 1970, los estudios sobre el fenómeno pretendían identificar cuál era el origen del mismo y obtener las características generales del proceso. Estas posturas pioneras tenían un enfoque reduccionista de lo que era ya el concepto de gentrificación, porque simplemente se basaban en las relaciones de causaefecto producidas por el fenómeno y abordaban el tema de una manera genérica, sólo contemplando el hecho de por qué se producía, y no todas las implicancias que traía aparejado. Por tanto, dicho concepto se utilizó básicamente para denominar los movimientos poblacionales que se producían en los barrios de clases obreras de la ciudad de Londres y cómo los nuevos habitantes de mayor poder adquisitivo se concentraban en la mejora y rehabilitación de las viviendas degradadas dejadas por sus antiguos pobladores.

Aparece años más tarde el concepto de "reconquista urbana" utilizado por Manuel Castells², el cual hacía referencia a los procesos que se habían emprendido en una serie de barrios parisinos, en los que planteaba que en realidad lo importante en el proceso era el cambio en la ocupación social del espacio, y no solamente significaba la mejora en sí de la vivienda o el barrio. Castells observaba cómo en estos movimientos de personas -con la consecuente transformación de las áreas afectadas-, aumentaba la segregación de las clases sociales de forma cada vez más pronunciada: los estratos superiores de la sociedad tendían a desplazarse hacia las áreas centrales, mientras las clases populares y de bajos recursos prácticamente se veían expulsadas hacia las zonas periféricas de la ciudad.

Luego de la década de los 70 , se dio un mayor desarrollo teórico del tema que posibilitó la ampliación de los contenidos del concepto, comenzando entonces a relacionarse el fenómeno con otros conceptos de naturaleza inherente a la ciudad, como pueden ser las condicionantes económicas, los parámetros sociales en que se desarrolla la misma, así también como las características espaciales que adopta.

Hacia mediados de la década de los 80 autores como Neil Smith ${ }^{3}$ ya señalaban la vinculación entre el proceso de gentrificación y la regeneración de algunas zonas de la ciudad abandonadas, ya que sus usos quedaron obsoletos dadas las nuevas dinámicas de las ciudades actuales. Como ejemplos podemos citar los frentes marítimos -que comenzaron a perfilarse para posibles usos recreativos y comerciales- así como también las instalaciones industriales de las áreas centrales de la ciudad que comenzaron a ser transformados en centros hoteleros, complejos de oficinas, modernas

1. GLASS, Ruth. Aspects of Change. University College London, Centre for Urban Studies. Londres: Macgibbon \& Kee

2. CASTELLS, Manuel. 1974. Planificación urbana y movimientos sociales: el caso de la renovación urbana de París. En: BERINGUIER, Christian; CASTELLS, Manuel; et al. Urbanismo y práctica política. Barcelona: Libros de la Frontera.

3. SMITH, Neil. 1979. Toward a Theory of Gentrification: A Back to the City Movement by Capital, not People. En: Journal of the American Planning Association. (45): (pp. 538-548) 
áreas comerciales y zonas gastronómicas de moda.

Durante estas primeras etapas de estudio del concepto de gentrificación surgieron distintas visiones o perspectivas del mismo tema. Unas posturas planteaban la gentrificación a partir de los cambios de las preferencias de consumo de los habitantes de la ciudad, situando de esta manera en primer plano el concepto de demanda. Otras explicaban la gentrificación a partir de la importancia del papel de los distintos agentes institucionales, tanto públicos como privados, que lo que buscaban era estimular la oferta inmobiliaria con el objetivo de obtener rentas elevadas de zonas donde se había producido total desinversión.

A mediados de la década de los 80 surge la necesidad de conciliar propuestas más integradas, no sólo basadas en los aspectos económicos o de demanda y oferta vigentes, sino también integrando aspectos sociales y agregándole contenidos culturales y de participación e interacción de las distintas clases sociales.

En la actualidad, el estudio de la gentrificación se centra en cómo se desarrolla, considerando cada caso en particular.

Para poder llevar a cabo este tipo de análisis, es necesario realizar estudios más detallados, para los cuales es fundamental tener en cuenta algunos de los siguientes aspectos:

- la historia de la ciudad en su conjunto y del origen del barrio en sí mismo,

- la planificación y el desarrollo urbano a los que se vio expuesta dicha zona,

- las particularidades existentes entre la zona potencialmente gentrificable y su relación con el entorno y el resto de la ciudad, incluyendo espacios públicos, arquitectura circundante, etc.,

- las características de los habitantes de la zona a través del tiempo.

Las nuevas posturas consideran al fenómeno como recíproco e interactuante, y centran su metodología de estudio básicamente en cómo se desarrolla la gentrificación, dejando su origen en un segundo plano de importancia.

En la actualidad para muchos de los autores la visión del contenido del término, está en directa relación con las oportunidades de consumo. El consumo ha dejado de ser una categoría residual, y por ello, las ciudades deben ser entendidas como áreas de consumo en sí mismas, lo cual toma vital importancia. Algunos autores como Mullins ${ }^{4}$-incluso a través de estudios de carácter empírico- proporcionan datos verídicos de cómo realmente existe una vinculación directa entre las áreas que él llamaría "elitizadas", con las áreas de gran concentración de espacios de consumo.

Los centros urbanos, que históricamente han sido los lugares de concentración de servicios, actividades y población, se han convertido justamente en los puntos focales para el desarrollo de la gentrificación.

La coexistencia de las distintas clases sociales dentro de la ciudad es una característica propia del concepto tradicional de la misma. Este aspecto ha experimentado transformaciones a lo largo de la evolución hacia la ciudad contemporánea, ligadas a los procesos de expansión y de movilización de la población.

El desarrollo de las nuevas tecnologías vinculadas al

4. MULLINS, P.; NATALIER, K.; SMITH, P. SMEATON, B. 1999. Cities and consumption spaces. En: Urban Affairs Review. (35): pp. 44-71. 
transporte y a la comunicación genera el comienzo de un nuevo ciclo de expansión de la ciudad. Un ejemplo de ello es cómo las industrias comienzan a ubicarse en las áreas periféricas de la ciudad, produciendo un traslado de la población ligada a esas actividades a áreas cercanas a aquéllas.

Esto marca una primera etapa de vaciamiento de las áreas centrales de la ciudad, quedando en ellas las actividades comerciales y sociales asociadas a las clases medias y altas, que aún no ven la necesidad de abandonar esta zona.

La creación de nuevos planes urbanísticos vinculados a los conceptos de mejora de calidad de vida, conjuntamente con la expansión de la ciudad, promueven las ventajas de vivir en los suburbios en contraposición con el residir en el centro de la ciudad, deteriorado y falto de políticas de planificación urbana. Con esto comienza una nueva etapa de vaciamiento ligado al traslado de las clases superiores fuera de los centros urbanos hacia estas nuevas áreas residenciales.

Con las sucesivas etapas de abandono comienza un proceso de desvalorización del suelo de las áreas centrales, lo que las convierte en un punto de atracción para la población de bajos recursos que no pertenece a ninguno de los sectores de la población original. Ésta se halla compuesta en su mayoría por trabajadores provenientes de las áreas rurales e inmigrantes en búsqueda de nuevas oportunidades.

El asentamiento de personas de bajos recursos en los centros urbanos lleva al deterioro de las infraestructuras y edificaciones de estas áreas, las cuales son utilizadas continuamente pero a su vez no son ni mantenidas ni renovadas.
A partir de las últimas décadas del siglo $X X$ surgen nuevas políticas, ideologías y preocupaciones acerca de la importancia de los antiguos centros urbanos. Con esto comienza un cambio en la concepción del patrimonio existente de las ciudades, a través de organizaciones como UNESCO u organizaciones locales, que hacen hincapié en la conservación de las áreas tradicionales de la ciudad ${ }^{5}$. Esto conlleva un proceso de promoción para la recuperación y recalificación de las mismas, que pueden ser impulsadas por autoridades gubernamentales, tanto nacionales como municipales, promotores privados y agentes inmobiliarios, o como iniciativa propia de algunos sectores de la población.

Las nuevas necesidades de la población pautan las formas de recuperación de estas áreas, incentivándose así la revalorización del suelo a través de la inclusión de nuevos usos en las antiguas áreas. Los esfuerzos se centran principalmente en actividades comerciales, culturales y recreativas que permitan luego fomentar el uso residencial.

Como consecuencia de esto, por un lado se incrementa el costo de vida en las áreas afectadas. Por otro lado, los ingresos de la población residente se mantienen, por lo cual se ven imposibilitados de permanecer en el área, lo que hace que éstos se vean obligados a abandonarla una vez que comienza la rehabilitación.

Este abandono puede darse de manera espontánea al enfrentar una imposibilidad económica, o por medio de incentivos provenientes de los nuevos interesados en que el área sea intervenida. También puede darse a través del abandono forzado cuando los residentes son arrendatarios u ocupantes ilegales de las propiedades a intervenir cuyo destino será modificado.

5. RIVIÈRE D'ARC, Hélène. 2005. Integralidad de las políticas urbanas de rehabilitación de centros históricos. En: Seminario-Taller Internacional “Desarrollo local en la recuperación de áreas centrales" Proyecto "Ciudad Vieja Renueva" Intendencia Municipal de Montevideo y Comisión Europea - Programa URB-AL. Montevideo. 


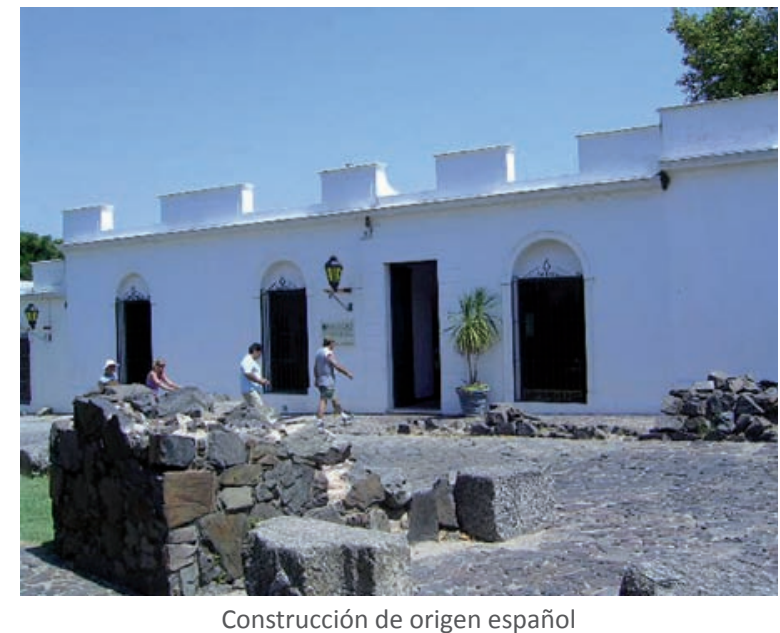

Los nuevos habitantes que comienzan a trasladarse a las áreas a intervenir pertenecen a un peldaño superior en la escala social, mayoritariamente jóvenes profesionales con ingresos relativamente elevados, lo cual les permite tener acceso a viviendas costosas.

Este estrato incluye distintos grupos sociales, tales como artistas, estudiantes, jubilados con ingresos altos, hogares monoparentales o incluso personas que viven solas, que debido básicamente a su posición económica, pueden también ser partícipes de este estilo de vida.

\section{Colonia del Sacramento}

Patrimonio, gentrificación e identidad

Las últimas décadas del siglo $X X$ se han visto caracterizadas por una revalorización del concepto de patrimonio, no sólo como bien material sino también como bien inmaterial. Esto se halla vinculado al proceso de internacionalización propio de la cultura contemporánea, que alude al sentimiento de nostalgia por parte de las personas con respecto al pasado propio y también con el ajeno, como forma de ligarse emocionalmente a distintas identidades culturales ${ }^{6}$.
Esto se manifiesta a través de lo que hoy se denomina "turismo cultural". Este tipo de turismo hace referencia al interés por los sitios de valor patrimonial, sean éstos de tipo natural o cultural.

La ciudad de Colonia del Sacramento adquirió trascendencia internacional cuando en 1995 su barrio histórico fue declarado Patrimonio Histórico de la Humanidad por la UNESCO, según el Criterio IV de selección ${ }^{7}$, por contar con testimonio notable en su trazado y construcciones, del asentamiento europeo colonial, de finales del siglo XVII. Esto trajo consigo el posicionamiento de la ciudad como uno de los puntos de atracción turística referente en la región.

El proceso que culminó luego en el reconocimiento por parte de la UNESCO, comenzó a fines de la década de los 60 con la figura del arquitecto Miguel Ángel Odriozola, quien llevó a cabo investigaciones acerca de los vestigios históricos en el casco antiguo y promovió la creación de la Comisión Honoraria del Patrimonio. Con el apoyo del Ministerio de Educación y Cultura, se comenzaron las excavaciones y posteriores intervenciones en las edificaciones portuguesas y españolas ${ }^{8}$.

6. BARRETO, Margarita. 2007. Turismo y Cultura: Relaciones, contradicciones y expectativas. Colección Pasos edita $\mathrm{N}^{\circ} 1$. Disponible en internet: www.pasosonline.org

7. “Ser un ejemplo sobresaliente de un tipo de construcción, de un conjunto arquitectónico o tecnológico, o de paisaje que ilustre una o más etapas significativas de la historia de la humanidad".

8. BARRETO. Margarita. 2007. Op. Cit. 


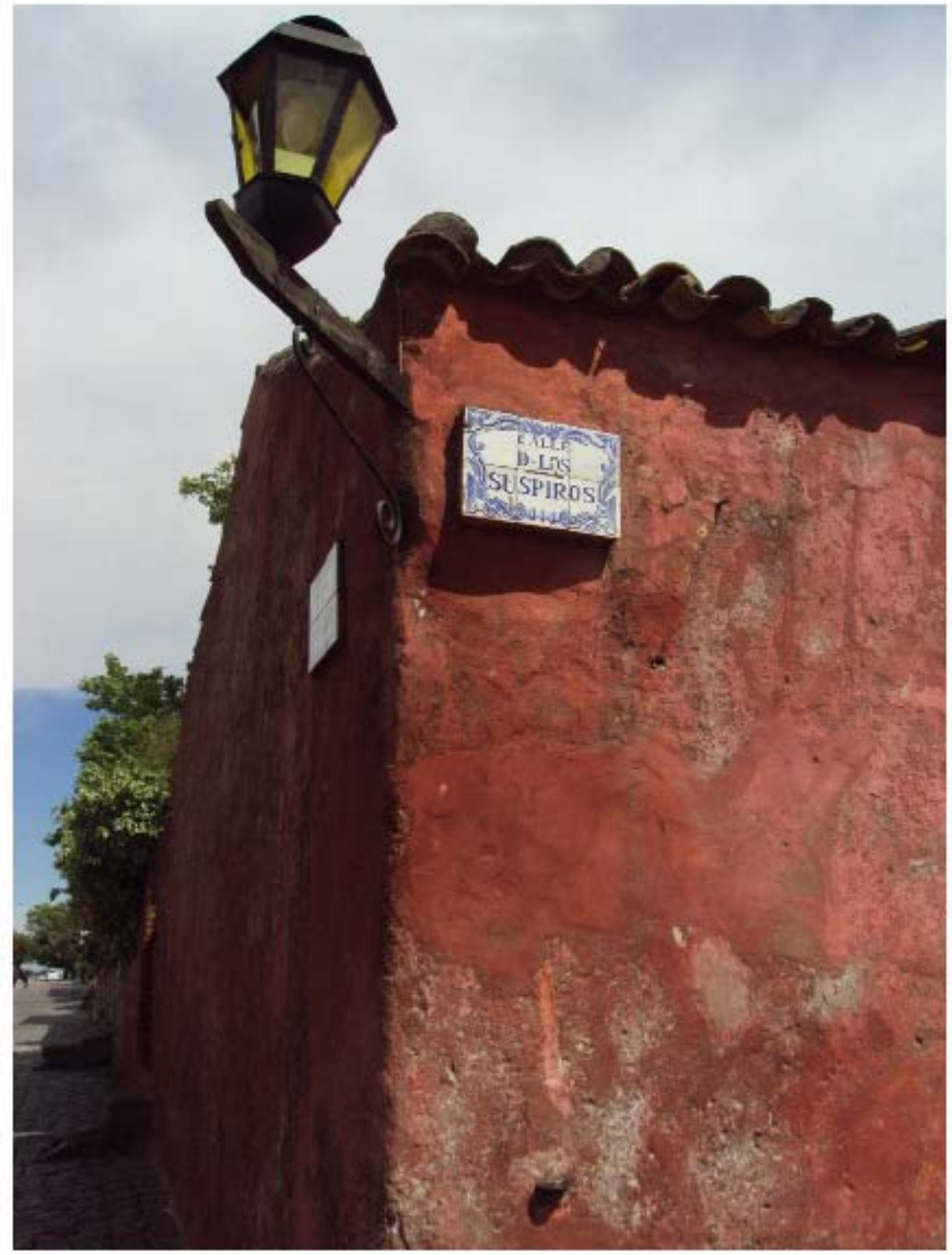

Construcción de origen portugués 

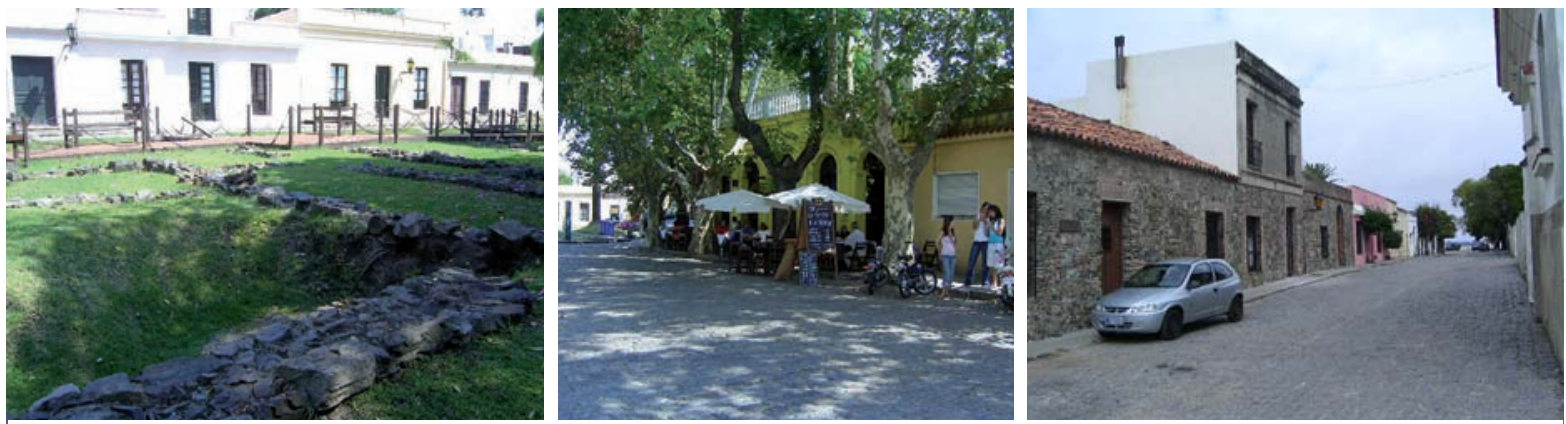

Reconstrucciones en barrio histórico

Las obras incluyeron la reconstrucción de la muralla que hasta 1968 no se encontraba visible, el Portón de Campo, el Museo Español, el Museo Portugués, los vestigios de la Casa del Virrey, las Ruinas del Convento, la Basílica entre otras edificaciones. Tales obras las cuales fueron finalizadas hacia 1972.

Las recomendaciones de la UNESCO pautaron la necesidad de proteger el patrimonio material, y para ello se estableció el Consejo Ejecutivo Honorario, con el cometido de regular todo tipo de intervención a realizarse en las edificaciones del área afectada. Sin embargo, la protección del patrimonio inmaterial no fue considerada bajo ninguna forma de regulación.

Por un lado, la valoración del sector se dio a nivel social a través del reconocimiento por parte de la población local, nacional e internacional, del valor real del sitio.

Por otro lado, esto implicó otro tipo de valoración referida al carácter administrativo, comercial y de los servicios propios de un centro urbano que lo convierten en un punto atractivo para la inversión. A finales de la década de los 80, muchas de las propiedades comienzan a ser adquiridas por capitales extranjeros, principalmente argentinos, que visualizan el potencial a futuro del sector.

Estos elementos marcan el comienzo de un proceso de transformación del antiguo Barrio Sur, el cual se había mantenido olvidado durante décadas mientras que la ciudad nueva se expandía en el área de extramuros. Tal como menciona Diego Blixen en su libro “De prostituta a señora." 9 , es en este nuevo Barrio Histórico en el que hasta hace treinta y pocos años vivieron en el olvido personas y personajes que se criaron entre la picaresca y la solidaridad, y que vieron mutar su propio barrio hacia el nuevo centro del turismo internacional.

Lentamente, los nuevos propietarios, propulsaron un ciclo de transformación de las antiguas construcciones para adecuarlas a las necesidades actuales a través de obras de rehabilitación, reconstrucción y reacondicionamiento, lo cual trajo aparejado el aumento en el valor de las mismas y del sector en general.

Como consecuencia directa del aumento en los costos de vida, los antiguos pobladores se vieron imposibilitados de permanecer en esa área, viéndose desplazados por residentes de mayor poder adquisitivo $^{10}$.

9. BLIXEN, Diego. De prostituta a señora: la historia reciente de Colonia del Sacramento. Ediciones del Caballo Perdido. 2005

10. GARCÍA MIRANDA, Ruben. Colonia del Sacramento. Aventuras y Desventuras de una Ciudad Histórica Iberoamericana. En: Seminario Internacional de Ciudades Históricas Iberoamericanas. [online] Toledo, 2001. [citado 18 de febrero de 2007] Disponible en Internet: http://www.esicomos.org/nueva_carpeta/ libroTOLEDO/22_rubengarciamiranda.html 

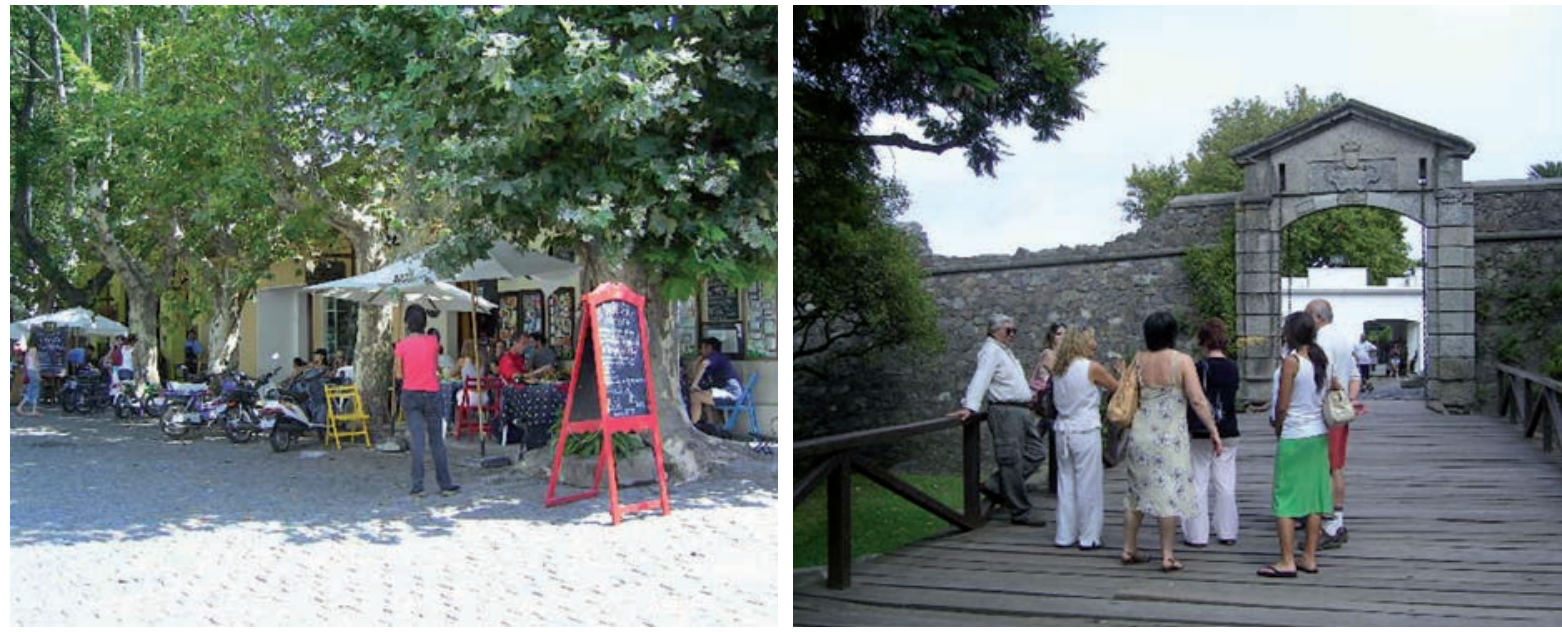

Actividad turística

Se corrobora entonces que, una vez más, el fenómeno de gentrificación no es ajeno a los procesos de revitalización, independientemente de las distintas escalas y coyunturas regionales, teniendo como principal implicancia la sustitución poblacional y el desplazamiento de la población original fuera del área revalorada.

El nuevo residente, conjuntamente con el nuevo tipo de usuario -el turista-, ambos de carácter temporal, generan nuevas dinámicas en el funcionamiento cotidiano del área.

El turismo es de perfil variado, pero siempre de visita corta, o como lo llaman los comerciantes locales, "de horario de barco", haciendo referencia a los horarios de llegada y salida de los barcos provenientes de Buenos Aires. Por lo tanto, existe un turista de alto nivel adquisitivo que permanece pocas horas en el lugar.

Se observa mayor intensidad de movimiento, sobre todo los fines de semana, que comienza con el arribo de los barcos en primeras horas de la mañana, aumenta en horas de la tarde, y luego desciende hacia la noche cuando parten los últimos barcos hacia Buenos Aires.

La aparición de servicios hoteleros, gastronómicos, recreativos, comerciales y financieros, que sustituyen gran parte del área anteriormente residencial, si bien procuran una revitalización del área también acentúan el carácter temporal de su uso. Esto ha traído como consecuencia la pérdida de la identidad, hasta ahora implícita, a través de sus bienes intangibles, los que desaparecieron junto con sus características de sector residencial y su población permanente.

Por todo esto, se puede afirmar que la ciudad de Colonia se ha transformado en una ciudad anfitriona, revalorizada, reconocida internacionalmente, abocada al turismo, atrayente de masas y capitales extranjeros, proveedora de servicios, foco cultural, elemento de consumo, ciudad contemporánea.

En definitiva, ciudad contemporánea, sin una identidad propia del lugar, de su historia y de su cultura, con identidad transformada, como un elemento al servicio del consumo. 


\section{BIBLIOGRAFÍA}

ACUÑA, Carlos; RIELLA, Alberto (comp.). 2003. Territorio, Sociedad y Región. Perspectivas desde el Desarrollo Regional y Local. Montevideo: Departamento de Sociología. Facultad de Ciencias Sociales, Universidad de la República.

AMENDOLA, Giandomenico. 2000. La Ciudad Postmoderna. Magia y Miedo de la Metrópolis Contemporánea. Madrid: Celeste.

BARRETO, Margarita. 2007. Turismo y Cultura: Relaciones, contradicciones y expectativas. Colección Pasos edita $\mathrm{N}^{\circ} 1$. Disponible en internet: www.pasosonline.org

BARRIOS PINTOS, Aníbal. 2000. Historia de los pueblos orientales. Tomo I. Montevideo: Libros de la Academia. BERINGUIER, C.; CASTELLS, Manuel, et al. 1974. Urbanismo y práctica política. Barcelona: Libros de la Frontera. BLIXEN, Diego. De prostituta a señora: la historia reciente de Colonia del Sacramento. Ediciones del Caballo Perdido. 2005

CAMPODÓNICO, Gabriela. Locales y Visitantes en Colonia del Sacramento. Un estudio de caso. [online] [citado 13 de diciembre 2006] Disponible en Internet: http://www.unesco.org.uy/shs/fileadmin/templates/ shs/archivos/anuario2004/articulo04_08.pdf

CASTELLS, Manuel. 1974. La cuestión urbana. Madrid: Siglo XXI de España.

CASTELLS, Manuel. 1976 (comp.). Estructura de clases y política urbana en América Latina. Madrid: Instituto de Estudio de Administración Local.

CRAVOTTO, Antonio. Aspectos urbanos y arquitectónicos. En: Colonia del Sacramento. Patrimonio Mundial. World Heritage. Montevideo: Testoni Studios Ediciones ; París: Ediciones UNESCO, c1996.

GAETA, Julio C.; FOLLE, Eduardo. 1997. Guías ElArqa de Arquitectura, 4. Colonia: Ciudad y Territorio. Montevideo: Dos Puntos.

GARCÍA CANCLINI, NÉSTOR. 1995. Consumidores y ciudadanos: conflictos multiculturales de la globalización. México: Grijalbo.

GARCÍA MIRANDA, Ruben. Colonia del Sacramento. Aventuras y Desventuras de una Ciudad Histórica Iberoamericana. En: Seminario Internacional de Ciudades Históricas Iberoamericanas. [online] Toledo, 2001. [citado 18 de febrero de 2007] Disponible en Internet: http://www.esicomos.org/nueva_carpeta/ libroTOLEDO/22_rubengarciamiranda.html

GLASS, Ruth. Aspects of Change. University College London, Centre for Urban Studies. Londres: Macgibbon \& Kee

INSTITUTO NACIONAL DE ESTADÍSTICA. 1996. VII Censo General de Población, III de Hogares y V de Viviendas: Colonia. Montevideo: Los Pinos.

JACOBS, Jane. 1961. The Death and Life of Great American Cities. Nueva York: Random House.

LEY, David. 1986. Alternative explanations for inner-city gentrification: A Canadian Assessment. En: Annals of the Association of American Geographers. (76): pp. 521 - 535.

LEY, David. 1994. Gentrification and the politics of the new middle class. En: Environment \& Planning D: Society \& Space. 12 (1): pp. 53-74. 


\section{BIBLIOGRAFÍA}

LOURÉS SEOANE, María Luisa. 2001. Del concepto de "monumento histórico" al de "patrimonio cultural". En: Ciencias Sociales. 1 (94): pp. 141-150.

MAYOR, Federico. Prefacio. En: Colonia del Sacramento. Patrimonio Mundial. World Heritage. Montevideo: Testoni Studios Ediciones ; París: Ediciones UNESCO, c1996.

MULLINS, P.; NATALIER, K.; SMITH, Ph.; SMEATON, B. 1999. Cities and consumption spaces. En: Urban Affairs Review. (35): pp. 44-71.

ODRIOZOLA, Miguel Ángel. 1990. El bastión del Carmen. En: Arquitectura (260). Montevideo: Sociedad de Arquitectos del Uruguay.

RED ACADÉMICA URUGUAYA. Ciudad de Colonia del Sacramento. [online] [citado 15 de diciembre de 2006]

Disponible en Internet: http://www.rau.edu.uy/uruguay/colonia

RIVIÈRE D'ARC, Hélène. 2005. Integralidad de las políticas urbanas de rehabilitación de centros históricos. En: Seminario-Taller Internacional "Desarrollo local en la recuperación de áreas centrales" Proyecto "Ciudad Vieja Renueva" Intendencia Municipal de Montevideo y Comisión Europea - Programa URB-AL. Montevideo.

SANTANA, Agustín. 1997. Antropología y turismo. ¿Nuevas hordas, viejas culturas? Barcelona: Ariel Antropología.

SARGATAL BATALLER, Ma Alba. 2000. El estudio de la gentrificación. En: Revista Bibliográfica de Geografía y Ciencias Sociales. (228).

SMITH, Neil. 1979. Toward a Theory of Gentrification: A Back to the City Movement by Capital, not People. En: Journal of the American Planning Association. (45): (pp. 538-548).

UNESCO. Convención para salvaguardar el patrimonio inmaterial: patrimonio vivo, patrimonio inmaterial. [online] [citado 2 de Abril 2007] Disponible en Internet: http://whc.unesco.org/archive/1995/whc-95-conf20316e.pdf

Fuente de ilustraciones: las fotografías han sido tomadas por las autoras

\section{Cómo citar:}

Nóbile, C., \& Sader, A. (2011). La identidad después de la gentrificación. Anales de Investigación en Arquitectura, 1(1), 49-60. https://doi.org/10.18861/ania.2011.1.1.3047 